\title{
Late-life depression in Rural China: do village infrastructure and availability of community resources matter?
}

\author{
Lydia W. $\mathrm{Li}^{1}$, Jinyu Liu ${ }^{1}$, Zhenmei Zhang ${ }^{2}$ and Hongwei $\mathrm{Xu}^{3}$ \\ ${ }^{1}$ University of Michigan, School of Social Work, Ann Arbor, MI, USA \\ ${ }^{2}$ Michigan State University, Department of Sociology, East Lansing, MI, USA \\ ${ }^{3}$ University of Michigan, Survey Research Center, Ann Arbor, MI, USA \\ Correspondence to: L. Li, PhD, E-mail: lydiali@umich.edu
}

\begin{abstract}
Objectives: This study aimed to examine whether physical infrastructure and availability of three types of community resources (old-age income support, healthcare facilities, and elder activity centers) in rural villages are associated with depressive symptoms among older adults in rural China.

Method: Data were from the 2011 baseline survey of the Chinese Health and Retirement Longitudinal Study (CHARLS). The sample included 3824 older adults aged 60 years or older residing in 301 rural villages across China. A score of 12 on the 10-item Center for Epidemiologic Studies Depression Scale was used as the cutoff for depressed versus not depressed. Village infrastructure was indicated by an index summing deficiency in six areas: drinking water, fuel, road, sewage, waste management, and toilet facilities. Three dichotomous variables indicated whether income support, healthcare facility, and elder activity center were available in the village. Respondents' demographic characteristics (age, gender, marital status, and living arrangements), health status (chronic conditions and physical disability), and socioeconomic status (education, support from children, health insurance, household luxury items, and housing quality) were covariates. Multilevel logistic regression was conducted.
\end{abstract}

Results: Controlling for individuals' socioeconomic status, health status, and demographic characteristics, village infrastructure deficiency was positively associated with the odds of being depressed among rural older Chinese, whereas the provision of income support and healthcare facilities in rural villages was associated with lower odds.

Conclusion: Village infrastructure and availability of community resources matter for depressive symptoms in rural older adults. Improving infrastructure, providing old-age income support, and establishing healthcare facilities in villages could be effective strategies to prevent late-life depression in rural China. Copyright (C) 2014 John Wiley \& Sons, Ltd.

Key words: neighborhood effects; infrastructure deficiency; old-age income support; rural older adults; depressive symptoms History: Received 16 May 2014; Accepted 3 September 2014; Published online 21 October 2014 in Wiley Online Library (wileyonlinelibrary.com)

DOI: 10.1002 /gps.4217

\section{Introduction}

Numerous studies have been conducted to understand factors related to late-life depression (Blazer, 2003; Fiske et al., 2009). Focus has primarily been on individual-level factors. More recently, there is increasing attention to the role of neighborhood context (Julien et al., 2012). Studies have shown that neighborhood socioeconomic disadvantages, usually indicated by the proportion of residents who live in poverty, are unemployed, and have low educational levels, were associated with depressive symptoms in older people in Western societies (Almeida et al., 2012; Kubzansky et al., 2005; Ostir et al., 2003). In this study, we investigated whether physical infrastructure and availability of community resources of rural villages are associated with depressive symptoms among older adults in rural areas of People's Republic of China (hereafter China). 


\section{Neighborhood context and depression in older adults}

In studying neighborhood effects on mental health, neighborhood features are often conceptualized as leading to either an adverse or supportive environment (Julien et al., 2012; Mair et al., 2008). An adverse environment increases individuals' exposure to stressors and experience of chronic strain, whereas a supportive environment boosts individuals' psychosocial resources and may act as buffers of stress (Mair et al., 2008; Paczkowski and Galea, 2010). Older adults' mental health may be particularly susceptible to the influence of neighborhood factors because of declining physical and cognitive capacity, reduced social support, and more time spent in the neighborhood (Yen et al., 2009).

\section{Study population}

This study focuses on older adults living in rural China. Previous studies have reported that this population has high rates of depressive symptoms and suicide, especially in comparison with their urban counterparts (Gao et al., 2009; Ma et al., 2008; Li et al., 2009; Zhang et al., 2012). Suicide rates among older Chinese in rural areas have been three to five times higher than those in urban areas (Li et al., 2009; Wang et al., 2014). Identifying neighborhood factors associated with depressive symptoms could shed new light on community-level interventions to prevent depression in this population. Such interventions have the potential to influence a large number of people (Julien et al., 2012).

Methodologically, focusing on rural older Chinese has two advantages. The first is reduced possibility of reverse causation. Reverse causation occurs when people with and without mental health problems move in and out of poor neighborhoods. China has a household registration system (hukou) which restricts individuals from moving to another location (Wang, 2005). Even though the restriction has loosened and domestic migration has accelerated in the past two decades (Huang and Pieke, 2003), the current cohort of rural older adults was too old to join the flow of migration. Second, rural village offers a delineation of neighborhood that likely corresponds to the life space of those living there, as rural residents live and work in the village where their hukou is. Most Western studies have used administrative boundaries such as zip code and census tract to define neighborhood, which has been criticized as not necessarily reflecting the neighborhood as perceived by residents (Julien et al., 2012).

Research on neighborhood context and mental health of older persons in China is scarce. A recent study reports that neighborhood identity is associated with mental health of older adults in Shanghai (Ye and Chen, 2014). Two studies report significant associations between neighborhood features and physical health outcomes among older persons in China. One suggests that environmental, economic, and social characteristics of rural villages in China are associated with physical function of middle-age and older adults (Yeatts et al., 2013). Another finds that neighborhood socioeconomic status (e.g., labor force participation) and physical environment (e.g., air pollution) predict physical disability and mortality in older Chinese (Zeng et al., 2010).

\section{Research questions and hypotheses}

Our study asked two questions. First, is village infrastructure associated with depressive symptoms among older adults living in rural China? Infrastructure here refers to basic physical structure (e.g., roads, drinking water supply, and sewer systems) needed for a community to function properly (Aschauer, 1989). The importance of infrastructure has often been discussed with reference to productivity and economic growth (Gramlich, 1994), but research has shown a linkage between infrastructure and child health (Thomas and Strauss, 1992). In China, local-level infrastructure is largely a local (sub-provincial) responsibility (Lin et al., 2003). China's economic growth in the past four decades has widened income inequalities between and within regions (Oizumi, 2010). Unequal wealth translates to unequal spending power among local governments. Relative to those living in villages with better infrastructure, older people living in villages with poor infrastructure may feel deprived, abandoned, or forgotten by those in power. The latter may also be more likely to feel incapable of changing or improving their living conditions, which may undermine a sense of control and efficacy (Hill and Maimon, 2013). Hence, we hypothesize that older people living in villages with more infrastructure deficiencies are more likely to be depressed.

Our second question asks: is availability of income support, healthcare facilities, and elder activity centers in the village associated with depressive symptoms of rural older Chinese? We chose to examine these three types of community resources because they may be particularly relevant to rural elders' mental health. Because of the under-development of public pension schemes in rural China, rural residents are vulnerable to poverty in old age (Cai et al., 2012). Living in villages that provide income support to their older residents should help to increase older people's sense of security 
and efficacy, and alleviate poverty-related stress. Having a healthcare facility in the village should facilitate older villagers' access to health care and lower the stress associated with health problems. Having an elder activity center in the village affords older people opportunities for social activities, which should help to reduce social isolation and increase social ties and support. Hence, we expect that older people living in rural villages are less likely to be depressed when their communities provide income support, a healthcare facility, and an elder activity center.

\section{Methods}

\section{Data and sample}

Data for this study were from the 2011 baseline survey of the China Health and Retirement Longitudinal Study (CHARLS), which interviewed a national representative sample of adults aged 45 years and older (main respondents) and their spouses if available. The sample was obtained through multistage cluster sampling, with a response rate of $80.5 \%$ (Zhao et al., 2013).

Our analysis included only rural respondents aged 60 years or older. Respondents were regarded as rural if they lived in neighborhoods governed by a village committee. If both the main respondent and his or her spouse were age eligible, only the main respondent was included. In addition to collecting individual-level data, the CHARLS conducted a community survey to collect data about the neighborhoods in which respondents resided through interviewing village leaders. The analyzed sample included 3824 respondents residing in 301 rural villages across China.

\section{Variables and measures}

Dependent variable. Our dependent variable was clinically significant depressive symptoms. It was measured by the 10-item Center for Epidemiologic Studies Depression Scale (CESD-10), which has been widely used in prior studies (Andresen et al., 1994) and validated in older Chinese in Hong Kong (Boey, 1999; Cheng and Chan, 2005). The sum of the CESD-10 scores ranges from 0 to 30 (Cronbach's alpha $=0.81$ ). Previous studies show that a score of 12 on the CESD-10 had reasonable levels of sensitivity (0.76) and specificity (0.55) among older persons in Hong Kong (Cheng and Chan, 2005; Cheng and Chan, 2008). Hence, we used 12 as the cutoff point to identify those who were depressed $(=1)$ and not depressed $(=0)$.
Neighborhood-level variables. We included four neighborhood-level variables in the analysis. The first variable, infrastructure deficiency, was assessed by an index that summed deficiency in six areas: drinking water, fuel, road, sewage, waste management, and toilet facilities. A score from 0 to 3 was assigned to each area, with 3 indicating the most deficient. Deficiency in drinking water and fuel was indicated by the proportion of households using tap water $(0$ was assigned if $100 \%, 1$ if $>50 \%$ and $<100 \%, 2$ if $>0 \%$ and $<50 \%$, and 3 if $0 \%)$ and hay $(0=0 \%, 1=>0 \%$ and $<50 \%$, $2=>50 \%$ and $<100 \%, 3=100 \%)$. Road deficiency was scored 0 if most roads in the village were paved, 2 if sand/stone, and 3 if unpaved. Regarding sewer system, a score of 0 was assigned if the village had such system and 3 if it did not. Waste management was assigned 0 if waste was moved away by trucks, 1 if it was buried in the village systematically, 2 if it was burned or put into nearby river systematically, and 3 if no management at all. Toilet facilities were scored 0 if the main type of toilet in the village was inside toilet with water, 1 if it was inside without water or outside with water, 2 if it was outside without water, and 3 if it was open-air. The infrastructure deficiency index ranged from 0 to 18; higher scores indicated more deficiencies (Cronbach's alpha $=0.74)$.

The second variable, old-age income support, was dichotomously coded $(1=$ yes vs. $0=$ no $)$ on the basis of a single question asking whether the village provided subsidy to residents older than 65 years. The third variable, health facility, was based on questions about eight types of healthcare facilities (e.g., general hospital, township health center, and village medical post). Health facility was coded $1=$ yes if at least one of these facilities was located in the village, and $0=$ no if none. The fourth variable, elder activity center, was coded $1=$ yes if the village had an elder activity center, otherwise coded $0=$ no.

Individual-leve/ variables. Demographic characteristics (age, gender, marital status, and living arrangements), socioeconomic status (SES) at the individual and household levels, and health status of respondents were used as covariates in the analysis. Age was measured in chronological years. Gender was dichotomously coded as female or male. Marital status was also coded dichotomously: currently married or not. Living arrangements had three mutually exclusive categories: empty nest (i.e., living alone or with spouse only), living with children, and living with someone other than children. 
Individual-level SES indicators included education (coded in three categories: illiterate, some schooling up to elementary, and junior high or more), health insurance (coded yes vs. no health insurance), and financial support from adult children (coded in four categories: none, low, medium, and high levels of support). 'None' was assigned to those who received no financial support from children. Among those who received support, we divided the total amount received in the previous year into tertiles, labeled as low, medium, and high levels of support. Income was not used because of limitations in both accuracy and measurement in the rural context (Deaton, 1997). To assess wealth or long-term living standard, we used household luxury items and housing quality (Bollen et al., 2002). Household luxury items were indicated by a count of 17 items (e.g., refrigerator, washing machine, and TV) that the household owned. Housing quality was measured by an index that summed quality of seven types of amenities (toilet, electricity, running water, shower/bath facility, fuel, phone, and internet connection) in the respondents' home (Cronbach's alpha $=0.60$ ). Each amenity was assigned a score from 1 to 3 ; for example, 3 was assigned if respondents had in-house flushable toilet, 2 if having in-house but not flushable toilet, and 1 if no in-house toilet. Higher scores on the index indicated better quality.

Health status of respondents was indicated by chronic conditions and physical disability. Chronic conditions were measured as the total number of diseases, from a list of 14, that respondents had been diagnosed with. Physical disability was measured by limitations in six activities of daily living (ADL such as dressing and bathing) and five instrumental activities of daily living (IADL such as preparing meals and taking medications). It was dichotomously coded (yes vs. no). No disability was defined as having no difficulty in all ADL and IADL items.

\section{Data analysis}

Because respondents were nested in neighborhoods and the dependent variable was measured dichotomously, we used multilevel logistic regression to analyze the data. Stata 13 was used in all analyses (StataCorp, 2013). The independent variables had modest amount of missing $(<1 \%)$, but the measure of depressive symptoms had about $14.5 \% \quad(n=553)$ missing. We conducted multiple imputation. Findings reported later combined results from analyses of five imputed datasets. We had repeated the same analysis excluding respondents with missing values on depressive symptoms and found a similar pattern of results as shown here.

\section{Results}

\section{Description of the sample}

Table 1 presents characteristics of the sample. Mean age of respondents was 68.62 years (range $=60-101$ ). There were slightly more men $(51.12 \%)$ than women (48.88\%). More than two-thirds were married (67.02\%). Most respondents were empty-nesters $(43.67 \%)$ or lived with their children (46.55\%); a minority $(9.78 \%)$ lived with people other than their children. About 11 percent $(10.96 \%)$ had junior high or more education, 44 percent (44.25\%) had schooling up to elementary level, and 45 percent (44.8\%) were illiterate. A vast majority (93.54\%) had health insurance. Almost half of the sample $(48.48 \%)$ received no financial support from children; $20.76 \%, 15.85 \%$, and $14.91 \%$, respectively, received low, medium, and high levels of support. On average, they had three luxury items in the household and a score of 12.52 on the housing quality index (range $=7-21$ ). They had on average 1.55 chronic conditions. A majority $(72.33 \%)$ had no physical disability. About 38 percent $(38.47 \%)$ were classified as depressed. On a bivariate level, respondents with and without depressive symptoms were different in all socio-demographic characteristics and health status at $p<0.05$.

The 301 villages in which respondents resided varied greatly in infrastructure deficiency, with a mean of 11.10 $(\mathrm{SD}=4.84$, range $=0-18)$ on the deficiency index. About a quarter $(24.25 \%)$ of the villages provided income support to their older residents. Eighty-one percent $(81.06 \%)$ had at least one healthcare facility, and 27 percent $(27.25 \%)$ had an elder activity center located in the village.

\section{Multilevel logistic regression models}

We first estimated a two-level logistic regression model with depressive symptoms as the outcome, individuallevel variables as predictors, and a random intercept at the neighborhood level. The results show that all covariates except age and health insurance were significantly correlated with depressive symptoms at $p<0.05$ (Model 1, Table 2). The odds of being depressed were higher for women than men, and for those who were not married than those who were married. Relative to those living alone or with their spouse only, rural older adults living with children and those living with others were more likely to be depressed. The odds of being depressed were higher for older persons who were illiterate and those with elementary education, compared with those with junior high or more education. Rural older adults receiving high levels of financial 
Table 1 Characteristics of respondents and villages in the sample

\begin{tabular}{|c|c|c|c|}
\hline & Total & Depressed (38.47\%) & Not depressed $(61.53 \%)$ \\
\hline \multicolumn{4}{|l|}{ Individual-level variables $(N=3824)$} \\
\hline $\operatorname{Age}^{\star \star \star}($ range $=60-101)$, mean $(S D)$ & $68.62(7.35)$ & $69.28(7.59)$ & $68.20(7.16)$ \\
\hline \multicolumn{4}{|l|}{ Gender $^{\star \star \star}(\%)$} \\
\hline Male & 51.12 & 41.26 & 57.29 \\
\hline Female & 48.88 & 58.74 & 42.71 \\
\hline \multicolumn{4}{|l|}{ Marital status ${ }^{\star \star \star}(\%)$} \\
\hline Married & 67.02 & 60.10 & 71.36 \\
\hline Not married & 32.98 & 39.90 & 28.64 \\
\hline \multicolumn{4}{|l|}{ Living arrangements $(\%)^{*}$} \\
\hline Empty nest & 43.67 & 41.54 & 45.01 \\
\hline With children & 46.55 & 47.04 & 46.24 \\
\hline With others & 9.78 & 11.42 & 8.75 \\
\hline \multicolumn{4}{|l|}{ Education $^{\star \star \star}(\%)$} \\
\hline Illiterate & 44.80 & 51.94 & 40.33 \\
\hline Elementary or less & 44.25 & 41.60 & 45.90 \\
\hline Junior high or more & 10.96 & 6.46 & 13.77 \\
\hline \multicolumn{4}{|l|}{ Health insurance $^{\star \star}(\%)$} \\
\hline Yes & 93.54 & 92.11 & 94.43 \\
\hline No & 6.46 & 7.89 & 5.57 \\
\hline \multicolumn{4}{|l|}{ Financial support from children ${ }^{\star \star \star}(\%)$} \\
\hline None & 48.48 & 47.72 & 48.96 \\
\hline Low & 20.76 & 23.25 & 19.21 \\
\hline Medium & 15.85 & 16.86 & 15.21 \\
\hline High & 14.91 & 12.17 & 16.62 \\
\hline Household luxury items ${ }^{\star \star \star}($ range $=0-12)$, mean $(S D)$ & $3.10(2.08)$ & $2.71(1.88)$ & $3.35(2.16)$ \\
\hline Housing quality ${ }^{\star \star \star}($ range $=7-21)$, mean $(S D)$ & $12.52(3.17)$ & $11.89(2.89)$ & $12.91(3.27)$ \\
\hline Chronic conditions $^{\star \star \star}($ range $=0-8)$ & 1.55 (1.42) & $2.00(1.56)$ & $1.27(1.24)$ \\
\hline \multicolumn{4}{|l|}{ Physical disability*^ (\%) } \\
\hline Yes & 27.67 & 43.37 & 17.85 \\
\hline No & 72.33 & 56.63 & 82.15 \\
\hline \multicolumn{4}{|l|}{ Neighborhood-level variables $(n=301)$} \\
\hline \multirow{2}{*}{\multicolumn{4}{|c|}{ Income support (\%) }} \\
\hline & & & \\
\hline Yes & 24.25 & & \\
\hline No & 76.75 & & \\
\hline \multicolumn{4}{|l|}{ Health facility (\%) } \\
\hline Yes & 81.06 & & \\
\hline No & 18.94 & & \\
\hline \multicolumn{4}{|l|}{ Elder activity center (\%) } \\
\hline Yes & 27.25 & & \\
\hline No & 76.75 & & \\
\hline
\end{tabular}

“"” indicates statistical significant differences between depressed and non-depressed groups. ${ }^{*} p<0.05 ;{ }^{* *} p<0.01 ;{ }^{* * *} p<0.001$.

support from adult children were less likely to be depressed than those receiving none. More household luxury items and better housing quality were associated with reduced risks of depression, whereas more chronic conditions and having physical disability were associated with increased risks.

Next, we added infrastructure deficiency index in the model (Model 2, Table 2). As shown, every one-point increase in the index was associated with $4 \%$ increase $(O R=1.040,95 \% C I=1.018,1.063)$ in the odds of being depressed, controlling for individuals' demographic characteristics, SES, and health status. We then tested the association of the three types of community resources with depressive symptoms. Model 3 (Table 2) shows that older adults living in villages that did not provide old-age income support had 28\% higher odds of being depressed than those living in villages that did $(O R=1.281,95 \% C I=1.010,1.625)$. The odds of being depressed for older adults living in villages that had no healthcare facility were $25 \%$ higher than the odds for those whose villages had a health facility $(O R=1.246$, $95 \% C I=0.993,1.564, p=0.057)$. Residence in villages with no elder activity center increased the likelihood of being depressed, but it was not significant at $p<0.10$ $(O R=1.188,95 \% C I=0.911,1.549)$.

\section{Discussion}

We found that controlling for individuals' SES, health status, and demographic characteristics, older Chinese 
Table 2 Multilevel logistic regression models for depressive symptoms in rural older Chinese: associations with individual-level and neighborhoodlevel variables ( $N=3824$ individuals, $n=301$ villages)

\begin{tabular}{|c|c|c|c|}
\hline & Model 1 & Model 2 & Model 3 \\
\hline & OR $(95 \% \mathrm{Cl})$ & OR $(95 \%$ Cl) & OR $(95 \% \mathrm{Cl})$ \\
\hline \multicolumn{4}{|c|}{ Individual-level independent variables } \\
\hline Age & $0.993(0.979,1.006)$ & $0.994(0.981,1.008)$ & $0.994(0.981,1.008)$ \\
\hline \multicolumn{4}{|l|}{ Gender (vs. male) } \\
\hline Female & $1.681(1.411,2.002)^{\star \star \star}$ & $1.703(1.429,2.029)^{\star \star \star}$ & $1.699(1.427,2.023)^{\star \star \star}$ \\
\hline \multicolumn{4}{|l|}{ Marital status (vs. married) } \\
\hline Not married & $1.282(1.069,1.538)^{\star \star}$ & $1.294(1.079,1.551)^{\star \star \star}$ & $1.296(1.081,1.553)^{\star \star}$ \\
\hline \multicolumn{4}{|c|}{ Living arrangements (vs. empty nest) } \\
\hline With children & $1.406(1.155,1.712)^{\star \star}$ & $1.373(1.128,1.672)^{\star \star}$ & $1.367(1.123,1.663)^{\star *}$ \\
\hline With others & $1.696(1.287,2.234)^{\star \star \star}$ & $1.637(1.242,2.157)^{\star \star \star}$ & $1.640(1.245,2.161)^{\star \star \star}$ \\
\hline \multicolumn{4}{|l|}{ Education (vs. junior high or more) } \\
\hline Illiterate & $1.491(1.099,2.022)^{\star}$ & $1.469(1.084,1.992)^{*}$ & $1.481(1.093,2.006)^{\star}$ \\
\hline Elementary or less & $1.542(1.159,2.051)^{\star \star}$ & $1.550(1.166,2.061)^{\star \star}$ & $1.558(1.173,2.069)^{\star \star}$ \\
\hline \multicolumn{4}{|l|}{ Health insurance (vs. yes) } \\
\hline No & $1.354(0.969,1.892)^{\dagger}$ & $1.366(0.977,1.909)^{\dagger}$ & $1.378(0.986,1.927)^{\dagger}$ \\
\hline \multicolumn{4}{|c|}{ Financial support from children (vs. none) } \\
\hline Low & $1.049(0.851,1.293)$ & $1.046(0.849,1.289)$ & $1.053(0.855,1.296)$ \\
\hline Medium & $1.027(0.800,1.318)$ & $1.036(0.808,1.329)$ & $1.040(0.811,1.334)$ \\
\hline High & $0.766(0.592,0.990)^{*}$ & $0.774(0.599,0.999)^{*}$ & $0.779(0.604,1.006)^{\dagger}$ \\
\hline Household luxury items & $0.871(0.827,0.918)^{\star \star \star}$ & $0.877(0.832,0.924)^{\star \star \star}$ & $0.879(0.834,0.926)^{\star \star \star}$ \\
\hline Housing quality & $0.938(0.910,0.968)^{\star \star \star}$ & $0.952(0.922,0.984)^{*}$ & $0.954(0.923,0.985)^{\star \star}$ \\
\hline Chronic conditions & $1.379(1.291,1.473)^{\star \star \star}$ & $1.370(1.283,1.464)^{\star \star \star}$ & $1.371(1.283,1.464)^{\star \star *}$ \\
\hline \multicolumn{4}{|l|}{ Physical disability (vs. no) } \\
\hline Yes & $2.722(2.270,3.264)^{\star \star \star}$ & $2.652(2.215,3.176)^{\star \star \star}$ & $2.652(2.217,3.174)^{\star \star \star}$ \\
\hline \multicolumn{4}{|c|}{ Neighborhood-level independent variables } \\
\hline Infrastructure deficiency & & $1.040(1.018,1.063)^{\star \star \star}$ & $1.029(1.004,1.055)^{\star}$ \\
\hline No income support (vs. yes) & & & $1.281(1.010,1.625)^{\star}$ \\
\hline No health facility (vs. yes) & & & $1.246(0.993,1.564)^{\dagger}$ \\
\hline No elder activity center (vs. yes) & & & $1.188(0.911,1.549)$ \\
\hline Neighborhood-level variance & $0.473(0.362,0.616)$ & $0.441(0.331,0.589)$ & $0.413(0.302,0.565)$ \\
\hline
\end{tabular}

$O R=$ odds ratio; $C I=$ confidence interval. ${ }^{\dagger} p<0.10 ;{ }^{*} p<0.05 ;{ }^{* *} p<0.01 ;{ }^{* * *} p<0.001$.

living in rural villages with poor infrastructure, no old-age income support, and no healthcare facility were more likely to be depressed than those living in villages that had better infrastructure, provided income support to their older residents, and had a healthcare facility located in the villages.

Multiple mechanisms, including psychological ones, may be responsible for the linkage between neighborhood infrastructure and depressive symptoms of rural older adults. Where individuals live has social significance and is tied to their self-concepts (Fitzpatrick and LaGory, 2011). In the context of China's rapid economic growth in recent decades, poor infrastructure of their villages may suggest to older residents that their villages are inferior to others and that they have been abandoned or forgotten by the government. Their self-worth may suffer as a consequence of negative social comparison and feelings of relative deprivation. They may also feel powerless to change their living conditions and lose hope about the future (Hill and Maimon, 2013). Additionally, poor infrastructure may undermine mental health of older adults by increasing environmental hazards and daily life stress. Lack of sewage and waste management systems, for example, may contribute to water pollution, which not only has negative health consequences but also increases negative emotions such as insecurity and anxiety due to restricted access to clean water.

Very few prior studies have investigated the role of neighborhood infrastructure in depression. Some studies in developed nations have reported that neighborhood socioeconomic disadvantages, indicated by demographic attributes (e.g., unemployment rates), are associated with depressive symptoms in older people (Almeida et al., 2012; Kubzansky et al., 2005; Ostir et al., 2003). Our results suggest that neighborhood infrastructure matters for depressive symptoms in rural older Chinese over and above individuals' SES and demographic characteristics, and that improving infrastructure of rural villages would be a preventive strategy of late-life depression in rural China.

Our findings also suggest that income support programs are potentially effective interventions to prevent depression in rural older Chinese. Our data collected 
in 2011 show that about $24 \%$ of the sampled villages provided old-age income support. The Chinese government has been pilot-testing a New Rural Pension Scheme since 2009 (Vilela, 2013). It seems that there is still a way to go to ensure income security in old age for rural residents. We also found that having a healthcare facility in villages reduced the risk of depression among older adults in rural China. About $81 \%$ of the villages in our sample had a healthcare facility. More than half $(55 \%)$ of them had a medical post, which is rudimentarily equipped and provides only very basic level care (Ding et al., 2013). However, even such basic healthcare facility could make a positive contribution to the mental health of rural older adults.

Several limitations of our study should be noted. First, it was based on cross-sectional data and cannot examine any causal relationships. We also cannot investigate the extent of exposure to neighborhood context in conjunction with the onset and trajectory of depressive symptoms. Second, we did not investigate mechanisms linking neighborhood features and the risk of depression. Longitudinal data are needed for such effort. Finally, our measures of neighborhoodlevel variables were based on reports of village leaders. The degree of accuracy and bias of these data is unclear.

This study has several strengths worth noting. It was based on a national probability sample of older residents in rural China, and had a relatively large sample size at both the individual and neighborhood levels. Further, the delineation of neighborhood in our study was likely to coincide with perceptions of those living there, given the administrative structure in rural China. Third, we examined neighborhood features that are theoretically relevant to older people's mental health but have not been investigated in prior studies. Fourth, we studied a population that was limited in residential mobility, reducing the possibility of reverse causation. In addition, our analysis has minimized compositional effects by controlling for an array of individual-level characteristics, including multiple indicators of SES and health status.

\section{Conclusion}

To conclude, our study has contributed to the understanding of neighborhood contextual influence in latelife depression in rural China, and identified some amendable village-level factors associated with the odds of being depressed among rural older Chinese. Supporting rural villages to improve physical infrastructure, provide old-age income support, and establish healthcare facilities could be effective strategies to prevent late-life depression in rural China.

\section{Conflict of interest}

None declared.

Key points

- Poor village infrastructure is significantly associated with higher odds of being depressed among older adults in rural China, controlling for individuals' SES, health status, and demographic characteristics.

- Older adults living in rural villages that provide old-age income support and have a healthcare facility are less likely to be depressed than their counterparts who live in villages that have no income support program and no healthcare facility.

- Improving infrastructure, providing old-age income support, and establishing healthcare facilities in rural villages could be effective strategies to reduce the prevalence of late-life depression in rural China.

\section{Acknowledgement}

Dr. Li was supported by a grant from the National Institute of Health (R01MH100298). Dr. Liu was supported by a post-doc fellowship at the Curtis Center, School of Social Work, University of Michigan. We thank the support provided by the Center for Statistical Consultation and Research at the University of Michigan.

\section{References}

\footnotetext{
Almeida OP, Pirkis J, Kerse N, et al. 2012. Socioeconomic disadvantage increases risk of prevalent and persistent depression in later life. J Affect Disord 138: 322-331. Andresen EM, Malmgren JA, Carter WB, Patrick DL. 1994. Screening for depression in well older adults: evaluation of a short form of the CES-D. Am J Prev Med 10: 77-84. Aschauer DA. 1989. Is public expenditure productive? J Monetary Econ 23: 177-200. Blazer DG. 2003. Depression in late life: review and commentary. J Gerontol Ser A-Biol Sci Med Sci 58: 249-265.

Boey KW. 1999. Cross-validation of a short form of the CES-D in Chinese elderly. Int J Geriatr Psychiatry 14: 608-617.

Bollen KA, Glanville JL, Stecklov G. 2002. Economic status proxies in studies of fertility in developing countries: does the measure matter? Popul Stud 56: 81-96.

Cai F, Giles J, O'Keefe P, Wang D. 2012. The Elderly and Old Age Support in Rural China. The World Bank: Washington DC.

Cheng ST, Chan A. 2005. The center for epidemiologic studies depression scale in older Chinese: thresholds for long and short forms. Int J Geriatr Psychiatry 20: 465-470.

Cheng ST, Chan A. 2008. Detecting depression in Chinese adults with mild dementia: findings with two versions of the Center for Epidemiologic Studies Depression Scale. Psychiatry Res 159: 44-49.

Deaton A. 1997. The Analysis of Household Surveys: A Microeconmic Approach to Development Policy. John Hopkins University Press: Baltimore.
} 
Ding Y, Smith HJ, Fei Y, et al. 2013. Factors influencing the provision of public health services by village doctors in Hubei and Jiangxi provinces, China. Bull World Health Organ 91: 64-69.

Fiske A, Wetherell JL, Gatz M. 2009. Depression in older adults. Annu Rev Clin Psychol 5: 363-389.

Fitzpatrick K, Lagory M. 2011. Unhealthy Cities: Poverty, Race, and Place in America. Routledge: New York.

Gao S, Jin Y, Unverzagt FW, et al. 2009. Correlates of depressive symptoms in rural elderly Chinese. Int J Geriatr Psychiatry 24: 1358-1366.

Gramlich EM. 1994. Infrastructure investment: a review essay. J Econ Lit 32: 1176-1196.

Hill TD, Maimon D. 2013. Neighborhood context and mental health. In Handbook of the Sociology of Mental Health. Springer: New York; 479-501.

Huang P, Pieke FN. 2003. China migration country study. Paper presented at the Regional Conference on Migration, Development and Pro-Poor Policy Choices in Asia. Dhaka, Bangladesh.

Julien D, Richard L, Gauvin L, Kestens Y. 2012. Neighborhood characteristics and depressive mood among older adults: an integrative review. Int Psychogeriatr 24: $1207-1225$.

Kubzansky LD, Subramanian SV, Kawachi I, et al. 2005. Neighborhood contextual influences on depressive symptoms in the elderly. Am J Epidemiol 162: 253-260.

Li X, Xiao Z, Xiao S. 2009. Suicide among the elderly in mainland China. Psychogeriatrics 9: 62-66.

Lin J, Tao R, Liu M. 2003. Decentralization and local governance in China's economic transition. Paper prepared for the conference: The Rise of Local Governments in Developing Countries. London School of Economics: London, UK.

Ma X, Xiang Y, Li S-R, et al. 2008. Prevalence and sociodemographic correlates of depression in an elderly population living with family members in Beijing, China Psychol Med 38: 1723-1730.

Mair C, Diez Roux AV, Galea S. 2008. Are neighbourhood characteristics associated with depressive symptoms? A review of evidence. J Epidemiol Community Health 62: 940-946.
Oizumi K. 2010. A geographical view of China's economic development—observations focusing on 337 prefecture-level cities. Pacific Bus Ind X: 2-32.

Ostir GV, Eschbach K, Markides KS, Goodwin JS. 2003. Neighbourhood composition and depressive symptoms among older Mexican Americans. J Epidemiol Community Health 57: 987-992.

Paczkowski MM, Galea S. 2010. Sociodemographic characteristics of the neighborhood and depressive symptoms. Curr Opin Psychiatry 23: 337-341.

StataCorp. 2013. Stata Statistical Software: Release 13. College Station, TX: StataCorp LP.

Thomas D, Strauss J. 1992. Prices, infrastructure, household characteristics and child height. J Dev Econ 39: 301-331.

Vilela A. 2013. Pension Coverage in China and the Expansion of the New Rural Social Pension. HelpAge International: London, UK.

Wang C-W, Chan CL, Yip PS. 2014. Suicide rates in China from 2002 to 2011: an update. Soc Psychiatr Psychiatr Epidemiol 49: 929-941.

Wang F. 2005. Organizing Through Division and Exclusion: China's Hukou System. Stanford University Press: Redwood City.

Ye MZ, Chen YW. 2014. The influence of domestic living arrangement and neighborhood identity on mental health among urban Chinese elders. Aging Ment Health 18: $40-50$.

Yeatts DE, Pei X, Cready CM, et al. 2013. Village characteristics and health of rural Chinese older adults: examining the CHARLS Pilot Study of a rich and poor province. Soc Sci Med 98: 71-78.

Yen IH, Michael YL, Perdue L. 2009. Neighborhood environment in studies of health of older adults: a systematic review. Am J Prev Med 37: 455-463.

Zeng Y, Gu D, Purser J, Hoenig H, Christakis N. 2010. Associations of environmental factors with elderly health and mortality in China. Am J Public Health 100: 298-305.

Zhang L, Xu Y, Nie H, Zhang Y, Wu Y. 2012. The prevalence of depressive symptoms among the older in China: a meta-analysis. Int J Geriatr Psychiatry 27: 900-906.

Zhao Y, Strauss J, Yang G, et al. 2013. China Health and Retirement Longitudinal Study -2011-2012 National Baseline Users' Guide. Peking University: Beijing. 